\title{
Controlling Adenoviral Replication to Induce Oncolytic Efficacy
}

\author{
Cristina Fillat $^{1,2, *}$, Anabel José ${ }^{1,2}$, Xavier Bofill-De Ros ${ }^{1,2}$, Ana Mato-Berciano ${ }^{1,2}$, Maria Victoria \\ Maliandi ${ }^{1,2}$ and Daniel Abate-Daga ${ }^{1,2, \#}$
}

\author{
${ }^{I}$ Programa Gens i Malaltia, Centre de Regulació Genòmica-CRG, UPF, Parc de Recerca Biomèdica de Barcelona- \\ PRBB, Spain \\ ${ }^{2}$ Centro de Investigación Biomédica en Red de Enfermedades Raras (CIBERER), Barcelona, Spain
}

\begin{abstract}
Over the last decade, cancer therapy has found itself challenged by the growing field of oncolytic virotherapy. Many different viruses are currently under study, investigating their potential to induce antitumor effects through repeated cycles of viral infection and cell lysis. It was, however, genetically-engineered replication-selective adenoviruses that were the first to enter clinical trials with cancer patients. The difficulties involved in combining selectivity and elevated potency in a single oncolytic adenovirus have led investigators to design and test many different approaches. Different strategies, based on the control of viral replication, are presented in the current review. We discuss how the growing knowledge of cell and tumour biology, with the advances made in adenoviral virology, has inspired the fine-tuning of genetically-engineered adenoviruses. Special emphasis is placed on the fundamentals behind the use of certain specific genetic elements, introduced into the viral genome to control viral gene expression and on describing the most important viral gene mutations.
\end{abstract}

Keywords: Adenovirus, oncolysis, cancer virotherapy, transcriptional targeting, post-transcriptional targeting, cancer gene therapy, insulators, tumor microenvironment.

\section{INTRODUCTION}

Among the multiple and diverse approaches to treating cancer, there is a common principle which consists of identifying the differential traits of normal and neoplasic cells that can eventually be targeted to induce a cytotoxic effect in tumour cells, without significantly affecting normal cells (Fig. 1). Said principle is equally applicable to adenoviral oncolytic strategies, most of which aim at restricting viral replication (and consequently cell lysis) to transformed cells. Early attempts involved the analysis of mutant forms of adenovirus, the replication of which is impaired in normal cells but not in tumour cells. More recently, with the development of recombinant DNA technology and the growing knowledge of molecular aspects of viral infection, tumour biology and gene regulation, a new generation of oncolytic adenovirus has been generated by genetically engineering viral genomes.

\section{ADENOVIRUSES INCORPORATING GENETIC ELEMENTS RESPONDING TO ALTERATIONS IN TUMOUR CELLS}

The complex regulatory mechanisms that control gene expression in mammalian cells, often deregulated in tumours, represent a toolbox full of tools that may be applied

\footnotetext{
*Address correspondence to this author at the Centre de Regulació Genòmica. Dr. Aiguader, 88. 08003-Barcelona, Spain; Tel: +34-933160142; E-mail: cristina.fillat@crg.es

${ }^{\#}$ Present address, Surgery Branch, National Cancer Institute, National Institutes of
} Health, Bethesda, MD, USA to the design of oncolytic adenoviruses. Transcriptional control, mRNA processing, mRNA stability and nuclear export, microRNA regulation and translational control all constitute processes from which the design of tumour selective adenovirus has benefited (Fig. 2).

\section{Transcriptional Targeting: Tumour-specific Promoters}

A large number of tumour-specific promoters that restrict adenoviral replication in tumour cells have been studied and a list of them, summarizing the principles and results, have been recently reviewed by Dorer and Nettelbeck [1]. In most of the studies only small portions of the natural promoters are used, and the given promoter fragment used may account for differences in promoter specificity. In general terms, the study of individual tumour-specific promoters (TSP) can be classified in two main groups: 1) promoters that can be considered pan-cancer specific, and will be of interest when targeting adenovirus to a variety of tumour types and 2) promoters that are tumour-type specific.

In the first category, the pan-cancer TSP, one of the most widely studied to date is human telomerase promoter. The promoter sequence was initially explored with a view to controlling viral replication, based on the fact that human telomerase is transcriptionally upregulated in about $90 \%$ of human cancers. hTERT controlled adenoviruses have been proven to provide tumour selectivity in models of cervical carcinoma [2], gastric [3], breast [4], head and neck and hepatocellular cancer [5], and non-small cell lung cancer [6]. hTERT promoter has been engineered in many different ways, controlling E1A [4], E1A and E1B [7], E4 [8] and in 


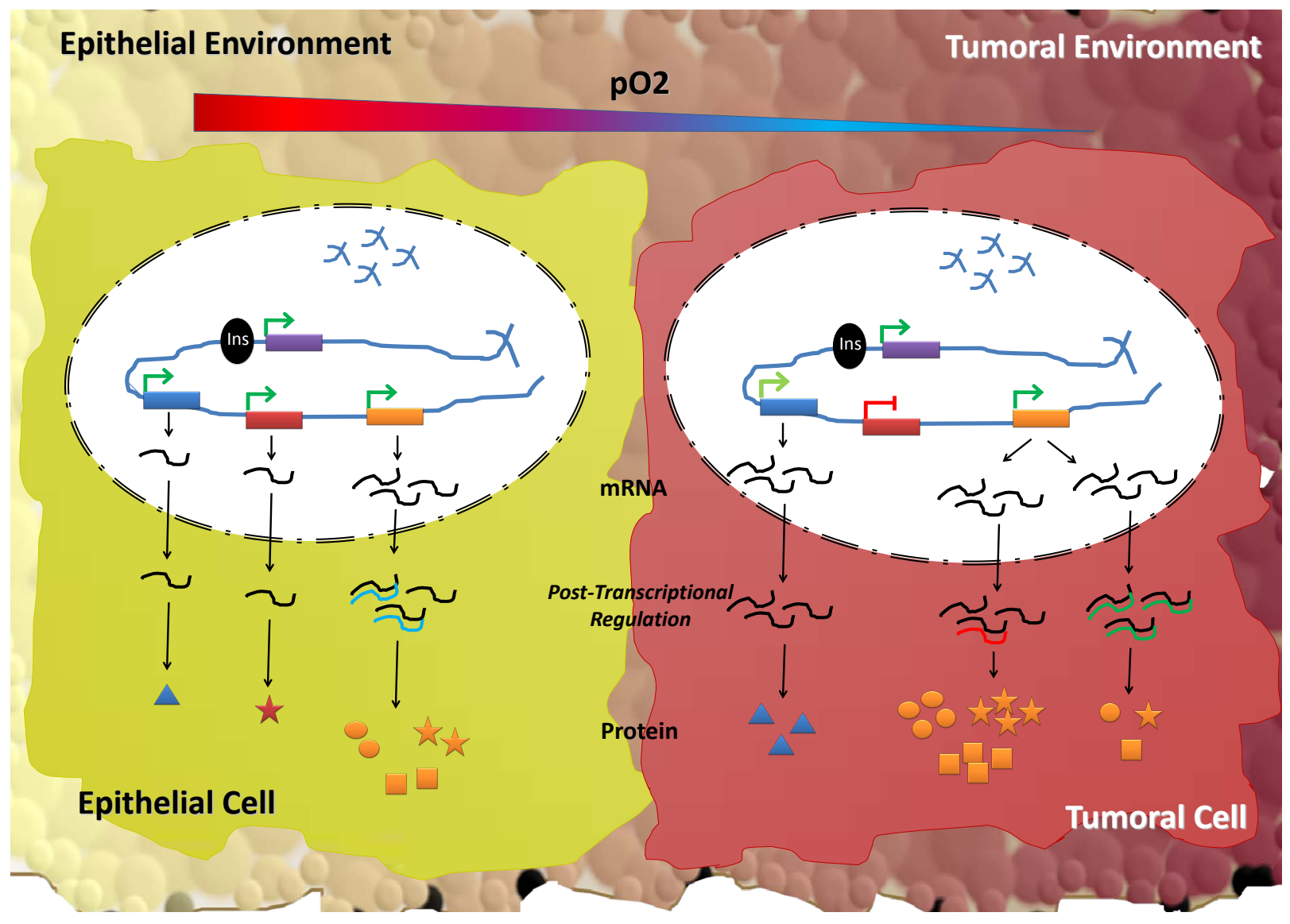

Fig. (1). Scheme representing molecular differences between epithelial and tumoral cells and their environment. In green it is shown the epithelial cell with genes and microRNAs (miR) expressed at normal levels. In red it is shown some of the molecular changes occurring in tumors: gene expression shut-off; gene up-regulation, miR deregulation either up or down. The diminished oxygen pressure pO2 is illustrating one of the major environmental changes in tumors.

a backbone armed with a variety of therapeutic genes [9]. The telomerase RNA promoter (hTR) has also been studied to selectively control replicating adenoviruses. Comparative studies between CRAds engineer with hTR and hTERT promoters contolling E1A have shown that despite achieving similar E1A levels hTR promoter was up to 49fold stronger than hTERT, evidencing feedaback regulation by E1A [10].

Another large group of studies have been generated exploring E2F transcription factor responsive promoters, working on the principle that almost every human malignancy involves mutations in the retinoblastoma tumour suppressor gene (RB1), or in components regulating the RB pathway that result in an excess of free E2F and a loss of $\mathrm{pRB} / \mathrm{E} 2 \mathrm{~F}$ repressor complexes. The fact that $\mathrm{E} 2 \mathrm{~F}$ is able to activate its own transcription has prompted the study of the E2F-1 promoter to control replication competent adenoviruses. E2F-1 oncolytic adenoviruses have shown tumour selectivity, efficacy and limited toxicity in many different cancer models [11-14]. Engineered E2F-1 E1A controlled in a backbone carrying viral gene mutations also targeting the $\mathrm{pRb}$ pathway (ex: $\mathrm{Ad} \Delta 24$, described in the viral gene mutation section) have shown antiglioma activity and reduced toxicity $[15,16]$. A modified E2F-1 promoter that incorporates additional palindrome $\mathrm{E} 2 \mathrm{~F}$ binding sites has recently been described and shown to improve the efficacy to toxicity ratio of oncolytic adenoviruses [17].
Another example is Cox-2 promoter-based replication selective adenoviruses. Cycloxygenase-2 (Cox-2) is an inducible enzyme in the biosynthetic pathway of prostaglandins that is over-expressed in a variety of premalignant and malignant conditions. Cox-2 controlled adenoviruses were first proposed by Yamamoto et al. to target pancreatic cancer [18]. However studies in many other tumour types, such as oesophageal, bladder. gallbladder, gastric and colorectal cancers, have been conducted and shown restricted replication and antineoplastic efficacy [19-23].

The survivin promoter is another well studied TSP. The rationale supporting the oncolytic use of the survivin promoter is based on the fact that survivin, the smallest member of the inhibitor of apoptosis family (IAPs), is transcriptionally upregulated in most human cancers, but not in normal tissue. Despite its potential general use to target many different types of tumours, survivin response oncolytic adenoviruses controlling E1A have mostly been studied in gliomas where they have shown oncolytic efficacy, either alone or in combination with other therapies [24-28]. A few studies have been conducted in other tumour models such as cholangiocarcinoma [29] and lung cancer [30].

Also other promoters, such as the receptor for chemokine SDF-1 known as CXCR4, upregulated in invasive cancers, have been studied for their selective targeting of adenoviruses to lung [31] and breast cancer [32], gliomas [24] or head and neck cancer [33]. 




Fig. (2). Genetically engineered strategies of the adenoviral genome to acquire oncolysis. Scheme of the adenoviral genome and highlighted in red the more common studied viral gene mutations.Tumor specific promoters (TSP) controlling the expression of viral genes with insulator elements, protecting from viral enhancer influences. Post-transcriptional targeting strategies: incorporation of selective 5'UTR or 3'UTR elements and the introduction of miR binding sites for target microRNAs.

Moreover, there are other oncolytic adenoviruses controlled by tumour selective promoters that have been studied in a single or very limited number of cancer models which have the potential to prove active in many other forms of tumour. One example is urokinase palsminogen activator receptor promoter (UPAR) controlled oncolytic adenoviruses which have recently been shown to present antitumor efficacy in pancreatic cancer models [34].

Also, the development of oncolytic adenovirus targeting cancer deregulated pathways such as the Wnt pathway have been studied. These oncolytic adenoviruses incorporate Tcf sites in early viral promoters and have shown to provide with selective replication in cells with activation of the Wntsignalling pathway $[35,36]$.

The second category of TSP controlled adenoviruses are those that use tumour type specific promoters. In fact in most of the cases this promoters are tissue-specific but they become highly activated in the tumor context, what will provide certain degree of tumor selectivity. Examples of this strategy are tyrosinase promoter to preferentially target melanoma cells [37, 38], alpha-fetoprotein [39] or trans- thyretin [40] for hepatocarcinoma, carcinoembryonic antigen (CEA) [41] or A33 antigen [42] for colorectal cancer, uroplakins specific for bladder cancer [43], glial fibrillary acidic protein (GFAP) promoter to target gliomas [44], mesothelyn promoter for ovarian cancer [45] or prostate specific antigen (PSA) selective adenoviruses for prostate cancer [46].

\section{Post-transcriptional Targeting}

\section{3'UTR Sequences}

The 3' untranslated region (UTR) of the messenger RNA plays an important role in the regulation of gene expression. Alterations to such regulatory sequences are often found in malignant cells and as we come to understand the mechanisms participating in the function of the elements of 3'UTR regulating gene expression we see the possibility of using them in the design of oncolytic adenoviruses. The expression of various tumour-associated proteins is enhanced in many tumours, in part due to the stabilization of the mRNA caused by the AU rich sequences of the 3'UTRs. The use of the 3'UTR of COX2 to regulate E1A gene expression has been 
shown to enable the tumour selective control of adenoviruses, based on the principle that activated RAS/P-MAPK will stabilize E1A mRNA containing COX-2 3'UTR sequences, allowing replication only in cells with activated RAS pathways [47]. More recently, Sarkioja et al. showed that the incorporation of AU-rich elements from the 3'UTR of COX-2 counteracts promoter induction in non-malignant cells but not in cancer cells, providing enhanced tumour selectivity to transgene expression [48]. The rationale of this combined approach was based on the, transcriptional activation of the Cox- 2 promoter in tumors and the differentially regulation of the $3^{\prime} \mathrm{UTR}$ of COX-2 in normal and malignant cells. In normal cells the activity of the promoter was abrogated by the degradation signal triggered by the 3'UTR, however in tumoral cells the stabilization of the 3' UTR, through the RAS pathways and the enhanced activation of the COX-2 promoter provided with increased selectivity to the virus.

\section{5'UTR Sequences}

Cancer specific mRNA translational control has been explored by the use of a 5' untranslated region (UTR) from Fibroblast Growth Factor 2 (FGF-2). The idea behind this approach is that translation initiation factors such as eIF4E are, in general, nearly always overexpressed in solid tumours and that the factor specifically binds to the cap structure of the mRNA during the first step of translation. Efficient translation will then take place in cells expressing high levels of eIF4E. The 5'UTR element from FGF-2 was chosen as it was known that in the presence of high eIF4E there was a drastic increase in the synthesis of FGF-2. The authors showed that the inclusion of said translational control mechanism lead to a significant improvement in tumour selectivity [49].

\section{microRNA Target Sites}

The discovery that small non-coding RNAs and, in particular, microRNAs are active players in cell gene regulation, alongside evidence showing that they are deregulated in tumoural cells, has brought a new paradigm to viral engineering. The pioneers in developing viral vectors that respond to endogenous microRNAs were Brown et al. [50]. These authors developed lentiviral vectors with target sites for a specific microRNA and showed that transgene expression was effectively suppressed in cell types expressing mir-142$3 \mathrm{p}$. The idea was further developed to control oncolytic virus selectivity, by limiting undesirable replication in normal cells [51-53]. Oncolytic adenoviruses have also been modified by this strategy to detarget adenoviral replication in hepatic cells, with the aim of reducing the liver toxicity associated with the systemic delivery of oncolytic adenoviruses. The incorporation of target sites for the highly abundant liver specific miR-122 in the 3'-untranslated region of the E1A gene resulted in a virus which showed attenuated replication in cells of hepatic origin while retaining full activity in cancer cells $[54,55]$.

\section{Targeting Cellular Pathways: Viral Gene Mutation}

One of the first conditionally replicative adenovirus (CRADs) to reach clinical trials, $d l 1520$ (ONYX-015 or $\mathrm{H} 101)$, carried a mutation in the E1B protein. It had previously been described that wild type E1B has the ability to inactivate $\mathrm{p} 53$, thus preventing the otherwise consequent cell cycle arrest and/or induction of apoptosis in response to infection [56]. It was therefore widely accepted that said specificity relied on the presence of a previously inactivated p53 pathway in the tumour cells, rendering them permissive to viral replication, while such replication would be blocked in normal cells by the activity of a functional p53 pathway [57]. Nevertheless, it was later proposed that the basis for the selectivity of $d l 1520$ was rather a defect in viral mRNA nuclear export in normal cells infected with E1b deficient viruses that was complemented in tumour cells, thus allowing for the correct synthesis of viral late-gene products and the assembly of virions [58] selectively in malignant cells.

Another differential trait that has been exploited as a source of tumour specificity is the higher replication rate of tumour cells. As part of their natural life cycle, adenoviruses utilize the biosynthetic machinery of the host cell to synthesize viral DNA and the other components needed to generate their progeny. The enzymes involved in these processes are upregulated in actively replicating cells during the S-phase of the cell cycle, and adenoviruses have evolved to induce entry of the infected cells in the S-phase. The process is mediated, in wild type adenovirus-infected cells, by the association of the viral protein E1a with the cellular protein $\mathrm{pRB}$, resulting in the dissociation of the $\mathrm{pRB}-\mathrm{E} 2 \mathrm{~F}$ complex which then leads to an increase in free E2F levels. Unbound E2F is in turn capable of activating the transcription of the genes involved in cell replication, thus mimicking a mitogenic signal [59]. Since adenoviral replication depends on E1a activity as an inductor of S-phase entry, adenoviruses with partial deletions in E1a have been assayed as tumour cell specific lytic agents. The approach is based both on the inability of mutant E1a to disrupt pRB-E2F complexes and on the presence of high levels of free transcriptionally active E2F as a common trait of most tumour cells. Thus while E1A-mutant adenoviruses are not able to induce S-phase entry in normal quiescent cells, and therefore not able to replicate and lyse host cells, they are able to replicate in actively proliferating cells. This principle has prompted the generation of mutant $\operatorname{Ad} \Delta 24$ [60-62] and $d l 922-947$ [63] adenoviruses, presenting deletions in the E1a protein of amino acids 121 to 128 and 122 to 129 , respectively, both encompassing the pRB binding domain. Another variant, also including the deletion of residues 4 to 25 of $\mathrm{E} 1 \mathrm{a}$, has been created to avoid its binding to $\mathrm{p} 300 / \mathrm{CBP}$, previously described to induce entry in the Sphase of normal cells [64].

A more sophisticated strategy has been designed around the differential response of normal and tumour cells to viral molecules. Protein Kinase R (PKR) has been described to phosphorylate the eukaryotic Initiation Factor 2 (eIF2) needed for initiation of translation. The phosphorylation has an inhibitory effect on eIF2 activity that, in the context of viral infections, acts as a functional barrier to viral replication by shutting down protein synthesis. Adenovirus, however, present a mechanism to circumvent this blockade that involves the synthesis of RNA molecules (termed ViralAssociated RNA, VA RNA) which interact with but do not activate PKR, inhibiting its activation by other double stranded viral RNAs and thus allowing for the synthesis of viral proteins [65]. The rationale for the application of this mechanism as a source of tumour selectivity stems from the 
observation that oncogenic Ras products can also prevent eIF2 activity [66] and therefore complement the lost function in adenoviruses in which the coding sequence for VA RNAs has been deleted $[67,68]$. The adenoviruses are expected to replicate in cells with activated Ras, which account for up to $80 \%$ of tumour cells [69], while their replication will be inhibited in normal cells by the activation of PKR.

\section{ENGINEERED VIRUS THAT RESPOND TO THE MICROENVIRONMENT OF THE TUMOUR}

Carcinogenesis and tumour progression is being considered not as a cell autonomous, cancer cell-centred condition, but rather as a disease involving complex heterotypic multicellular interactions. The microenvironment of the tumour has been recognized as an integrated and essential part of the cancer tissue developing crosstalk between different cell types [70, 71]. Critical stromal elements include cancerassociated fibroblasts, which provide an essential communication network via the secretion of growth factors and chemokines by inducing an altered Extracellular Matrix (ECM) and thus providing additional oncogenic signals which enhance cancer cell proliferation and invasion. The microenvironment of cancer cells also provide the signals needed to turn on the transcription programs that influence tumourogenesis and metastasis [72]. Rapidly growing tumours quickly result in a poorly vascularised microenvironment, characterized by hypoxia, low $\mathrm{pH}$, and nutrient starvation $[73,74]$. Such environmental stress also plays a key role in regulating tumour progression from micro-invasiveness to metastatic cancer [75].

The knowledge of the microenvironment of the tumour thus gained has also been incorporated when designing transgene expression modulation to create tumour selective adenoviruses by means of different approaches.

1) Oncolytic adenoviruses responding to tumour stress: A well studied approach is that of controlling the E1a gene or, in a few cases, the E1b gene, under promoter sequences susceptible to activation when subject to hypoxia. Under low oxygen tumour cells stabilize the transcription factor HIF-1 which mediates transcriptional activation by binding to Hypoxia Responsive Elements (HRE). This has led to the generation of oncolytic adenoviruses that contain synthetic optimized promoters bearing multiple HRE or endogenous promoters, such as the hTERT, or the VEGF promoters containing HRE sequences. These viruses have been shown to replicate and efficiently kill tumour cells when subjected to hypoxia [76-84].

2) Oncolytic adenoviruses target cells in the microenviroment: Cell-specific promoters to control E1A targeted to dividing endothelial cells has been studied as an antiangiogenic approach. Regulatory elements of Flk-1 and endoglin genes were used to construct CRADs which strikingly inhibited the formation of a capillary network [85].

Several oncolytic adenovirus have also been generated to target both tumoural and tumour-associated stromal cells by using specific promoters. These have shown a significant level of oncolytic activity, with examples of complete remission of the tumour in certain cases. Examples include onco- lytic adenoviruses controlled by osteocalcin [86]. SPARC [87], or UPAR promoters [34]. Another gene promoter that has been much studied and is active both in malignant cells and in specific stromal cell types is COX-2. Examples of the oncolytic efficacy of COX-2 promoter based CRADs have been reported for several tumour models, such as pancreatic, oesophageal, gastric, colorectal, gallbladder, glioblastoma or cervical cancer $[14,18,21-23,88]$. The COX-2 promoter finds itself tightly regulated in response to inflammation and accordingly, recent research has been carried out into the role that anti-inflammatory drugs could exert on Cox-2 dependent viral replication and has shown that they may attenuate viral replication [88]. This is a factor that should be borne in mind since the treatment of cancer patients with anti-inflammatory molecules, such as dexamethasone, may end up compromising oncolytic efficacy. On the other hand, it may also find applications as a way of controlling potential undesired viral dissemination.

Co-targeting approaches to tumour, stromal and endothelial cells have been explored by engineering adenovirus which control E1A with FLK-1 and osteocalcin promoters. Said investigation has shown that combined therapy resulted in the regression of $30 \%$ of pre-established tumours [89].

\section{FINE-CONTROL OF GENE EXPRESSION IN ENGINEERED VIRUS. LEARNING FROM THE ARCHITECTURE OF THE GENOME}

The genetic engineering of the adenoviral genome has evidenced the fact that the introduction of heterologous promoters to control viral gene expression does not fully maintain the activity of endogenous genes since the latter are often affected by other neighbouring regulatory elements present in the adenoviral genome, resulting in interference and the consequent loss of expression selectivity [90-94]. Adenoviral promoters contain multiple recognition sites for transcription factors, often present as cis-acting DNA sequences, which increase transcription in a manner that is independent of their orientation and distance relative to the RNA start site. The presence of viral enhancers and upstream regulatory elements in the vector genome might interfere with heterologous promoters to drive transgene expression. Hoffman et al. identified an enhancer element within the Ad5 (nt 1-353) that could interact with and modulate some but not all heterologous promoters, suggesting that the specificity of a given promoter in an adenoviral context should be individually tested [95].

In order to develop a general design that would avoid such interference, researchers started to look at the general rules that preserve gene expression in the mammalian genome and made the observation that in the cell, throughout evolution, certain DNA sequences have been recruited by the genome to serve as boundaries to adequately insulate gene expression.

There are two classes of such insulators, enhancer blocking (EB) insulators, which prevent distal enhancers from activating a promoter when placed between the enhancer and promoter, and barrier insulators, which block heterochromatinization and the consequent silencing of a gene [96]. The barrier function of an insulator may protect a chromatin domain from the non-specific effects of the surrounding 
chromatin by blocking the passage of the regulatory signals from adjacent loci and chromatin domains [97, 98]. Their ability to guard against position effects is an interesting feature which is being exploited in integrative vectors.

Several such insulator elements have been studied, with a view to preserving the fidelity of newly introduced regulatory sequences in the adenoviral genome and ensure regulated expression. The insulator most studied in vertebrate genomes is the 5' end of the chicken beta-globin locus, associated with a constitutive DNAse I hyper- sensitive site, 5'HS4 [99]. It has been reported that the chicken $\beta$-globin locus insulator is a complex element with separable enhancer-blocking and position-effect functions (barrier function) [100]. The enhancer-blocking insulation activity of the 5' end of the chicken $\beta$-globin locus, as is the case in practically all vertebrate insulators found to date, is based on the binding of the zinc-finger protein CCCTC-binding factor (CTCF) that mediates the inhibition of promoter-enhancer interactions [101]. CTCF, a ubiquitously-expressed 11-zinc finger protein, is a critical transcription factor involved in transcriptional activation and repression, in addition to binding chromatin insulators [102-104]. Said insulator element was employed to shield an inducible promoter from viral enhancers and tested with first-generation adenoviral vectors in vitro and in vivo. Optimal shielding was obtained when the transgene expression cassette was flanked on both sides by HS-4 elements. The data provided the first evidence that insulators could exert enhancer-blocking effects in episomal DNA [94, 105]. The insulator effect of HS4, however, has been reported to be lost at an increased viral dose (100 MOI) [106]. This phenomenon could be explained by the fact that the expression cassette remains slightly leaky despite the presence of the insulator elements with a cumulative leakage present at increased viral MOI. In a recent study Rhomer and co-workers showed that the chicken beta-globin insulator preserves the heat-inducibility of the hsp70B promoter in replication-defective adenoviruses and clearly improves induction, when compared to its non-insulated counterpart. However, in replication-competent adenoviruses the regulation of the hsp70B promoter was lost during late replication and could not be rescued by insulator elements [107].

The insulating activity of the myotonic dystropy locus insulator (DM-1) was tested, bearing in mind the fact that it carries two binding sites for CTCF, is of human origin and is compact. The insulator was cloned between the E1a enhancer and E2F-1 promoter to improve the fidelity of the human tumour-selective E2F-1 promoter in previously described virus AdD24RGD [108]. Higher levels of E1A expression and viral production were recorded in tumour cell lines, as compared to normal cells, without any significant loss of antitumoural efficacy. This indicates that the presence of the DM-1 insulator conferred high promoter fidelity [109]. Another insulator element that has been shown to protect promoter activity in the context of oncolytic adenoviruses is the H19 insulator. A prostate specific promoter insulated with the $1.6 \mathrm{~Kb}$ core $\mathrm{H} 19$ insulator showed promising results for the treatment of localized prostate cancer [110].

Based on the current data the impact of insulators, with respect to preserving the fidelity of specific promoters in the context of oncolytic adenoviruses, remains controversial and is not yet fully understood, and may well be found to depend on the particular characteristics of a given promoter. However, in some cases, when large size insulators are used, this can compromise vector titres and the stability of viral particles [111]. Since, in general, the reduction in vector titers roughly correlates to the size of the insert [112], minimal size insulator sequences should be employed to avoid interference with viral production.

\section{CONCLUSIONS}

The therapeutic application of adenoviral oncolysis has evolved from the earliest attempts, which used mutant adenovirus generated by sustained culture in tumour cells, to today's sophisticated genetically engineered viruses designed to target and destroy disease-related cells in a tumour specific fashion [113]. The versatility of adenoviruses, in terms of backbone design and the production of high titre viral stocks, alongside their high efficiency transduction, have positioned them in the avant-garde of advanced medical approaches to the treatment of cancer.

Understanding the basic mechanisms that regulate cancer cell behaviour and their differences with respect to normal cells, has been instrumental in the design of oncolytic adenoviruses. The recent discovery of the new regulatory phenomenon by which small non-coding double stranded RNA molecules, and in particular microRNAs, can mediate the expression of hundreds of target genes has opened up the possibility of engineering the viral genome to be microRNA responsive. This approach is rapidly emerging as a versatile new targeting strategy and further exploitation of it may prove of great benefit with respect to the targeting of oncolytic adenoviruses. Likewise, the increased knowledge and greater control of the microenvironment of the tumour is proving as important as the knowledge and control of the transformed cancer cell when devising novel oncolytic designs [114].

To date, the field has mostly concentrated on developing viruses that efficiently and selectively replicate in tumours. But by applying similar principles future efforts are likely to focus on the study of the host-adenovirus interaction, in terms of the immunological response to viral particles and to virus-infected tumour cells. By viewing the immune system as a partner, rather than the enemy, it should be possible to develop a new generation of oncolytic adenoviruses with great potential as anticancer agents.

\section{ACKNOWLEDGEMENTS}

Research in the authors' laboratory was supported by the Spanish Ministry of Science and Innovation, BIO200804692-C03-02, and received partial support from the Generalitat de Catalunya SGR091527. A. José and X. Bofill are supported by a fellowship (FPU) from the Spanish Ministry of Science and Innovation. D Abate-Daga was a recipient of a Fundación Carolina fellowship. C. Fillat's group is also partially funded by CIBERER, Instituto de Salud Carlos III.

\section{REFERENCES}

[1] Dorer DE, Nettelbeck DM. Targeting cancer by transcriptional control in cancer gene therapy and viral oncolysis. Adv Drug Deliv Rev 2009; 61(7-8): 554-71.

[2] Kim E, Kim JH, Shin HY, et al. Ad-mTERT-delta19, a conditional replication-competent adenovirus driven by the human telomerase 
promoter, selectively replicates in and elicits cytopathic effect in a cancer cell-specific manner. Hum Gene Ther 2003; 14(15): 141528.

[3] Zhang Q, Nie M, Sham J, et al. Effective gene-viral therapy for telomerase-positive cancers by selective replicative-competent adenovirus combining with endostatin gene. Cancer Res 2004; 64(15): 5390-7.

[4] Zou W, Luo C, Zhang Z, et al. A novel oncolytic adenovirus targeting to telomerase activity in tumor cells with potent. Oncogene 2004; 23(2): 457-64.

[5] Lei N, Shen FB, Chang JH, et al. An oncolytic adenovirus expressing granulocyte macrophage colony-stimulating factor shows improved specificity and efficacy for treating human solid tumors. Cancer Gene Ther 2009; 16(1): 33-43.

[6] Dong F, Wang L, Davis JJ, et al. Eliminating established tumor in nu/nu nude mice by a tumor necrosis factor-alpha-related apoptosis-inducing ligand-armed oncolytic adenovirus. Clin Cancer Res 2006; 12(17): 5224-30.

[7] Taki M, Kagawa S, Nishizaki M, et al. Enhanced oncolysis by a tropism-modified telomerase-specific replication-selective adenoviral agent OBP-405 ('Telomelysin-RGD'). Oncogene 2005; 24(19): 3130-40.

[8] Kuppuswamy M, Spencer JF, Doronin K, et al. Oncolytic adenovirus that overproduces ADP and replicates selectively in tumors due to hTERT promoter-regulated E4 gene expression. Gene Ther 2005; 12(22): 1608-17.

[9] Cody JJ, Douglas JT. Armed replicating adenoviruses for cancer virotherapy. Cancer Gene Ther 2009; 16(6): 473-88.

[10] Bilsland AE, Merron A, Vassaux G, Keith WN. Modulation of telomerase promoter tumor selectivity in the context of oncolytic adenoviruses. Cancer Res 2007; 67(3): 1299-307.

[11] Tsukuda K, Wiewrodt R, Molnar-Kimber K, Jovanovic VP, Amin KM. An E2F-responsive replication-selective adenovirus targeted to the defective cell cycle in cancer cells: potent antitumoral efficacy but no toxicity to normal cell. Cancer Res 2002; 62(12): 343847.

[12] Ryan PC, Jakubczak JL, Stewart DA, et al. Antitumor efficacy and tumor-selective replication with a single intravenous injection of OAS403, an oncolytic adenovirus dependent on two prevalent alterations in human cancer. Cancer Gene Ther 2004; 11(8): 555-69.

[13] Li Y, Idamakanti N, Arroyo T, et al. Dual promoter-controlled oncolytic adenovirus CG5757 has strong tumor selectivity and significant antitumor efficacy in preclinical models. Clin Cancer Res 2005; 11(24 Pt 1): 8845-55.

[14] Hoffmann D, Meyer B, Wildner O. Improved glioblastoma treatment with Ad5/35 fiber chimeric conditionally replicating adenoviruses. J Gene Med 2007; 9(9): 764-78.

[15] Alonso MM, Cascallo M, Gomez-Manzano C, et al. ICOVIR-5 shows E2F1 addiction and potent antiglioma effect in vivo. Cancer Res 2007; 67(17): 8255-63.

[16] Cascallo M, Alonso MM, Rojas JJ, et al. Systemic toxicity-efficacy profile of ICOVIR-5, a potent and selective oncolytic adenovirus based on the pRB pathway. Mol Ther 2007;15(9): 1607-15.

[17] Rojas JJ, Cascallo M, Guedan S, et al. A modified E2F-1 promoter improves the efficacy to toxicity ratio of oncolytic adenoviruses. Gene Ther 2009; 16(12): 1441-51.

[18] Yamamoto M, Davydova J, Wang M, et al. Infectivity enhanced, cyclooxygenase-2 promoter-based conditionally replicative adenovirus for pancreatic cancer. Gastroenterology 2003; 125(4): 120318.

[19] Davydova J, Le LP, Gavrikova T, et al. Infectivity-enhanced cyclooxygenase-2-based conditionally replicative adenoviruses for esophageal adenocarcinoma treatment. Cancer Res 2004; 64(12): 4319-27.

[20] Shirakawa T, Hamada K, Zhang Z, et al. A cox-2 promoter-based replication-selective adenoviral vector to target the cox-2expressing human bladder cancer cells. Clin Cancer Res 2004;10(13): 4342-8.

[21] Ono HA, Davydova JG, Adachi Y, et al. Promoter-controlled infectivity-enhanced conditionally replicative adenoviral vectors for the treatment of gastric cancer. J Gastroenterol 2005; 40(1): 3142.

[22] Tekant Y, Davydova J, Ramirez PJ, et al. Oncolytic adenoviral therapy in gallbladder carcinoma. Surgery 2005; 137(5): 527-35.

[23] Hoffmann D, Wildner O. Restriction of adenoviral replication to the transcriptional intersection of two different promoters for colorectal and pancreatic cancer treatment. Mol Cancer Ther 2006; 5(2): 374-81.

[24] Ulasov IV, Rivera AA, Sonabend AM, et al. Comparative evaluation of survivin, midkine and CXCR4 promoters for transcriptional targeting of glioma gene therapy. Cancer Biol Ther 2007; 6(5): 679-85.

[25] Ulasov IV, Zhu ZB, Tyler MA, et al. Survivin-driven and fiber-modified oncolytic adenovirus exhibits potent antitumor activity in established intracranial glioma. Hum Gene Ther 2007; 18(7): 589-602.

[26] Nandi S, Ulasov IV, Tyler MA, et al. Low-dose radiation enhances survivin-mediated virotherapy against malignant glioma stem cells. Cancer Res 2008; 68(14): 5778-84.

[27] Tyler MA, Ulasov IV, Sonabend AM, et al. Neural stem cells target intracranial glioma to deliver an oncolytic adenovirus in vivo. Gene Ther 2009; 16(2): 262-78.

[28] Ulasov IV, Sonabend AM, Nandi S, et al. Combination of adenoviral virotherapy and temozolomide chemotherapy eradicates malignant glioma through autophagic and apoptotic cell death in vivo. Br J Cancer 2009; 100(7): 1154-64.

[29] Zhu ZB, Chen Y, Makhija SK, et al. Survivin promoter-based conditionally replicative adenoviruses target cholangiocarcinoma. Int J Oncol 2006; 29(5): 1319-29.

[30] Li B, Liu X, Fan J, et al. A survivin-mediated oncolytic adenovirus induces non-apoptotic cell death in lung cancer cells and shows antitumoral potential in vivo. J Gene Med 2006; 8(10): 123242.

[31] Zhu ZB, Rivera AA, Makhija SK, et al. Targeting lung cancer using an infectivity enhanced CXCR4-CRAd. Lung Cancer 2007; 55(2): $145-56$

[32] Stoff-Khalili MA, Rivera AA, Stoff A, et al. Combining high selectivity of replication via CXCR4 promoter with fiber chimerism for effective adenoviral oncolysis in breast cancer. Int J Cancer 2007; 120(4): 935-41.

[33] Zhu ZB, Mathis JM, Makhija SK, et al. Targeting of a conditionally replicative adenovirus agent to human squamous cell carcinomas of the head and neck. Int J Oncol 2007; 31(5): 1213-22.

[34] Huch M, Gros A, Jose A, et al. Urokinase-type plasminogen activator receptor transcriptionally controlled adenoviruses eradicate pancreatic tumors and liver metastasis in mouse models. Neoplasia 2009; 11(6): 518-28, 4 p following 28.

[35] Homicsko K, Lukashev A, Iggo RD. RAD001 (everolimus) improves the efficacy of replicating adenoviruses that target colon cancer. Cancer Res 2005; 65(15): 6882-90.

[36] Peerlinck I, Merron A, Baril P, et al. Targeted radionuclide therapy using a Wnt-targeted replicating adenovirus encoding the $\mathrm{Na} / \mathrm{I}$ symporter. Clin Cancer Res 2009; 15(21): 6595-601.

[37] Zhang L, Akbulut H, Tang Y, et al. Adenoviral vectors with E1A regulated by tumor-specific promoters are selectively cytolytic for breast cancer and melanoma. Mol Ther 2002; 6(3): 386-93.

[38] Banerjee NS, Rivera AA, Wang M, et al. Analyses of melanomatargeted oncolytic adenoviruses with tyrosinase enhancer/promoterdriven E1A, E4, or both in submerged cells and organotypic cultures. Mol Cancer Ther 2004; 3(4): 437-49.

[39] Sagawa T, Yamada Y, Takahashi M, et al. Treatment of hepatocellular carcinoma by AdAFPep/rep, AdAFPep/p53, and 5fluorouracil in mice. Hepatology 2008; 48(3): 828-40.

[40] Hsieh JL, Lee CH, Teo ML, et al. Transthyretin-driven oncolytic adenovirus suppresses tumor growth in orthotopic and ascites models of hepatocellular carcinoma. Cancer Sci 2009; 100(3): 53745.

[41] Li Y, Chen Y, Dilley J, et al. Carcinoembryonic antigen-producing cell-specific oncolytic adenovirus, OV798, for colorectal cancer therapy. Mol Cancer Ther 2003; 2(10): 1003-9.

[42] Cafferata EG, Maccio DR, Lopez MV, et al. A novel A33 promoter-based conditionally replicative adenovirus suppresses tumor growth and eradicates hepatic metastases in human colon cancer models. Clin Cancer Res 2009; 15(9): 3037-49.

[43] Zhang J, Ramesh N, Chen Y, et al. Identification of human uroplakin II promoter and its use in the construction of CG8840, a urothelium-specific adenovirus variant that eliminates established bladder tumors in combination with docetaxel. Cancer Res 2002; 62(13): 3743-50

[44] Horst M, Brouwer E, Verwijnen S, et al. Targeting malignant gliomas with a glial fibrillary acidic protein (GFAP)-selective oncolytic adenovirus. J Gene Med 2007; 9(12): 1071-9. 
[45] Tsuruta Y, Pereboeva L, Breidenbach M, et al. A fiber-modified mesothelin promoter-based conditionally replicating adenovirus for treatment of ovarian cancer. Clin Cancer Res 2008; 14(11): 3582-8.

[46] DeWeese TL, van der Poel H, Li S, et al. A phase I trial of CV706, a replication-competent, PSA selective oncolytic adenovirus, for the treatment of locally recurrent prostate cancer following radiation therapy. Cancer Res 2001; 61(20): 7464-72.

[47] Ahmed A, Thompson J, Emiliusen L, et al. A conditionally replicating adenovirus targeted to tumor cells through activated RAS/PMAPK-selective mRNA stabilization. Nat Biotechnol 2003; 21(7): 771-7.

[48] Särkioja M, Hakkarainen $\mathrm{T}$, Eriksson $\mathrm{M}$, et al. The cyclo-oxygenase 2 promoter is induced in nontarget cells following adenovirus infection, but an AU-rich 3prime-untranslated region destabilization element can increase specificity. Gene Med 2008; 10(7): 744-53.

[49] Stoff-Khalili M, Rivera A, Nedeljkovic-Kurepa A, et al. Cancerspecific targeting of a conditionally replicative adenovirus using mRNA translational control. Breast Cancer Res Treatment 2008; 108(1): 43-55.

[50] Brown BD, Venneri MA, Zingale A, Sergi LS, Naldini L. Endogenous microRNA regulation suppresses transgene expression in hematopoietic lineages and enables stable gene transfer. Nat Med 2006; 12(5): 585-91.

[51] Edge RE, Falls TJ, Brown CW, et al. A let-7 MicroRNA-sensitive vesicular stomatitis virus demonstrates tumor-specific replication. Mol Ther 2008; 16(8): 1437-43.

[52] Kelly EJ, Hadac EM, Greiner S, Russell SJ. Engineering microRNA responsiveness to decrease virus pathogenicity. Nat Med 2008; 14(11): 1278-83.

[53] Lee CYF, Rennie PS, Jia WWG. MicroRNA Regulation of Oncolytic Herpes Simplex Virus-1 for Selective Killing of Prostate Cancer Cells. Clin Cancer Res 2009; 15(16): 5126-35.

[54] Ylosmaki E, Hakkarainen T, Hemminki A, et al. Generation of a conditionally replicating adenovirus based on targeted destruction of E1A mRNA by a cell type-specific MicroRNA. J Virol 2008; 82(22): 11009-15.

[55] Cawood R, Chen HH, Carroll F, et al. Use of tissue-specific microrna to control pathology of wild-type adenovirus without attenuation of its ability to kill cancer cells. PLoS Pathog 2009; 5(5): e1000440.

[56] Barker DD, Berk AJ. Adenovirus proteins from both E1B reading frames are required for transformation of rodent cells by viral infection and DNA transfection. Virology 1987; 156(1): 107-21.

[57] Bischoff JR, Kirn DH, Williams A, et al. An adenovirus mutant that replicates selectively in p53-deficient human tumor cells. Science 1996; 274(5286): 373-6.

[58] O'Shea CC, Johnson L, Bagus B, et al. Late viral RNA export, rather than p53 inactivation, determines ONYX-015 tumor selectivity. Cancer Cell 2004; 6(6): 611-23.

[59] Bandara LR, La Thangue NB. Adenovirus E1a prevents the retinoblastoma gene product from complexing with a cellular transcription factor. Nature 1991; 351(6326): 494-7.

[60] Fueyo J, Gomez-Manzano C, Alemany R, et al. A mutant oncolytic adenovirus targeting the $\mathrm{Rb}$ pathway produces anti-glioma effect in vivo. Oncogene 2000; 19(1): 2-12.

[61] Suzuki K, Fueyo J, Krasnykh V, et al. A conditionally replicative adenovirus with enhanced infectivity shows improved oncolytic potency. Clin Cancer Res 2001; 7(1): 120-6.

[62] Bauerschmitz GJ, Lam JT, Kanerva A, et al. Treatment of ovarian cancer with a tropism modified oncolytic adenovirus. Cancer Res 2002; 62(5): 1266-70.

[63] Heise C, Hermiston T, Johnson L, et al. An adenovirus E1A mutant that demonstrates potent and selective systemic anti-tumoral efficacy. Nat Med 2000; 6(10): 1134-9.

[64] Howe JA, Mymryk JS, Egan C, Branton PE, Bayley ST. Retinoblastoma growth suppressor and a 300-kDa protein appear to regulate cellular DNA synthesis. Proc Natl Acad Sci USA 1990; 87(15): 5883-7.

[65] Kitajewski J, Schneider RJ, Safer B, et al. Adenovirus VAI RNA antagonizes the antiviral action of interferon by preventing activation of the interferon-induced eIF-2 alpha kinase. Cell 1986; 45(2): 195-200.

[66] Mundschau LJ, Faller DV. Oncogenic ras induces an inhibitor of double-stranded RNA-dependent eukaryotic initiation factor 2 alpha-kinase activation. J Biol Chem 1992; 267(32): 23092-8.
[67] Cascallo M, Capella G, Mazo A, Alemany R. Ras-dependent oncolysis with an adenovirus VAI mutant. Cancer Res 2003; 63(17): 5544-50.

[68] Cascallo M, Gros A, Bayo N, et al. Deletion of VAI and VAII RNA genes in the design of oncolytic adenoviruses. Hum Gene Ther 2006; 17(9): 929-40.

[69] Macaluso M, Russo G, Cinti C, et al. Ras family genes: an interesting link between cell cycle and cancer. J Cell Physiol 2002; 192(2): 125-30.

[70] Bissell MJ, Radisky DC, Rizki A, Weaver VM, Petersen OW. The organizing principle: microenvironmental influences in the normal and malignant breast. Differentiation 2002; 70(9-10): 537-46.

[71] Hanahan D, Weinberg RA. The hallmarks of cancer. Cell 2000; 100(1): $57-70$

[72] Weinberg R. The biology of cancer. Garland Science. New York Taylor \& Francis Group, LLC 2007.

[73] Denko NC. Hypoxia, HIF1 and glucose metabolism in the solid tumour. Nat Rev Cancer 2008; 8(9): 705-13.

[74] Pouyssegur J, Dayan F, Mazure NM. Hypoxia signalling in cancer and approaches to enforce tumour regression. Nature 2006; 441(7092): 437-43.

[75] Ruan K, Song G, Ouyang G. Role of hypoxia in the hallmarks of human cancer. J Cell Biochem 2009; 107(6): 1053-62.

[76] Hernandez-Alcoceba R, Pihalja M, Qian D, Clarke MF. New oncolytic adenoviruses with hypoxia- and estrogen receptor-regulated replication. Hum Gene Ther 2002; 13(14): 1737-50.

[77] Cuevas Y, Hernandez-Alcoceba R, Aragones J, et al. Specific oncolytic effect of a new hypoxia-inducible factor-dependent replicative adenovirus on von Hippel-Lindau-defective renal cell carcinomas. Cancer Res 2003; 63(20): 6877-84.

[78] Post DE, Van Meir EG. A novel hypoxia-inducible factor (HIF) activated oncolytic adenovirus for cancer therapy. Oncogene 2003; 22(14): 2065-72.

[79] Cho WK, Seong YR, Lee YH, et al. Oncolytic effects of adenovirus mutant capable of replicating in hypoxic and normoxic regions of solid tumor. Mol Ther 2004; 10(5): 938-49.

[80] Irving J, Wang Z, Powell S, et al. Conditionally replicative adenovirus driven by the human telomerase promoter provides broad-spectrum antitumor activity without liver toxicity. Cancer Gene Ther 2004; 11(3): 174-85.

[81] Lam JT, Kanerva A, Bauerschmitz GJ, et al. Inter-patient variation in efficacy of five oncolytic adenovirus candidates for ovarian cancer therapy. J Gene Med 2004; 6(12): 1333-42.

[82] Takayama K, Reynolds PN, Adachi Y, et al. Vascular endothelial growth factor promoter-based conditionally replicative adenoviruses for pan-carcinoma application. Cancer Gene Ther 2007; 14(1): 105-16.

[83] Wang X, Su C, Cao H, et al. A novel triple-regulated oncolytic adenovirus carrying p53 gene exerts potent antitumor efficacy on common human solid cancers. Mol Cancer Ther 2008; 7(6): 1598603 .

[84] Bortolanza S, Bunuales M, Otano I, et al. Treatment of Pancreatic Cancer With an Oncolytic Adenovirus Expressing Interleukin-12 in Syrian Hamsters. Mol Ther 2009; 17(4): 614-22.

[85] Savontaus MJ, Sauter BV, Huang TG, Woo SL. Transcriptional targeting of conditionally replicating adenovirus to dividing endothelial cells. Gene Ther 2002; 9(14): 972-9.

[86] Hemminki A, Kanerva A, Kremer EJ, et al. A canine conditionally replicating adenovirus for evaluating oncolytic virotherapy in a syngeneic animal model. Mol Ther 2003; 7(2): 163-73.

[87] Lopez M, Viale D, Cafferata E, et al. Tumor associated stromal cells play a critical role on the outcome of the oncolytic efficacy of conditionally replicative adenoviruses. PLoS One 2009; 4(4): e5119.

[88] Kanerva A, Lavilla-Alonso S, Raki M, et al. Systemic therapy for cervical cancer with potentially regulatable oncolytic adenoviruses. PLoS One 2008; 3(8): e2917.

[89] Jin F, Xie Z, Kuo CJ, Chung LW, Hsieh CL. Cotargeting tumor and tumor endothelium effectively inhibits the growth of human prostate cancer in adenovirus-mediated antiangiogenesis and oncolysis combination therapy. Cancer Gene Ther 2005; 12(3): 257-67.

[90] Hatfield L, Hearing P. Redundant elements in the adenovirus type 5 inverted terminal repeat promote bidirectional transcription in vitro and are important for virus growth in vivo. Virology 1991; 184(1): 265-76. 
[91] Leza MA, Hearing P. Cellular Transcription Factor Binds to Adenovirus Early Region Promoters and to a Cyclic AMP Response Element. J Virol 1988; 3003-13.

[92] Ring C, Harris J, Hurst H, Lemoine N. Suicide gene expression induced in tumour cells transduced with recombinant adenoviral, retroviral and plasmid vectors containing the ERBB2 promoter. Gene Ther 1996; 3(12): 1094-103.

[93] Shi Q, Wang Y, Worton R. Modulation of the specificity and activity of a cellular promoter in an adenoviral vector. Hum Gene Ther 1997; 8(4): 403-10.

[94] Steinwaerder D, Lieber A. Insulation from viral transcriptional regulatory elements improves inducible transgene expression from adenovirus vectors in vitro and in vivo. Gene Ther 2000; 7: 556-67.

[95] Hoffmann D, Jogler C, Wildner O. Effects of the Ad5 upstream E1 region and gene products on heterologous promoters. J Gene Med 2005; 7(10): 1356-66.

[96] Wallace JA, Felsenfeld G. We gather together: insulators and genome organization. Curr Opin Genet Dev 2007; 15(5): 400-7.

[97] Burgess-Beusse B, Farrell C, Gaszner M, et al. The insulation of genes from external enhancers and silencing chromatin. Proc Natl Acad Sci USA 2002; 99(4): 16433-7.

[98] Razin S, Farrell C, Recillas-Targa F. Genomic domains and regulatory elements operating at the domain level. Int Rev Cytol 2003; 226: 63-125.

[99] Chung J, Whiteley M, Felsenfeld G. A 5' element of the chicken betaglobin domain serves as an insulator in human erythroid cells and protects against position effect in Drosophila. Cell 1993; 74(3): 50514.

[100] Recillas-Targa F, Pikaart MJ, Burgess-Beusse B, et al. Position-effect protection and enhancer blocking by the chicken -globin insulator are separable activities. Proc Natl Acad Sci USA 2002; 99: 6883-8.

[101] West A, Gaszner M, Felsenfeld G. Insulators: many functions, many mechanisms. Genes Dev 2002; 16(3): 271-88.

[102] Gaszner M, Felsenfeld G. Insulators: exploiting transcriptional and epigenetic mechanisms. Nat Rev Genet 2006; 7(9): 703-13.

[103] Ohlsson R, Renkawitz R, Lobanenkov V. CTCF is a uniquely versatile transcription regulator linked to epigenetics and disease. Trends Genet 2001; 17(9): 520-7.
[104] Williams A, Flavell R. The role of CTCF in regulating nuclear organization. J Exp Med 2008; 205(4): 747-50.

[105] Ye X, Liang M, Meng X, et al. Insulation from viral transcriptional regulatory elements enables improvement to hepatoma-specific gene expression from adenovirus vectors. Biochem Biophys Res Commun 2003; 307: 759-64.

[106] Martin-Duque P, Jezzard S, Kaftansis L, Vassaux G. Direct comparison of the insulating properties of two genetic elements in an adenoviral vector containing two different expression cassettes. Hum Gene Ther 2004; 15(10): 995-1002.

[107] Rohmer S, Mainka A, Knippertz I, Hesse A, Nettelbeck DM. Insulated hsp70B! promoter: stringent heat-inducible activity in replication-deficient, but not replication-competent adenoviruses. J Gene Med 2008; 10: 340-54

[108] Suzuki K, Fueyo J, Krasnykh V, et al. A conditionally replicative adenovirus with enhanced infectivity shows improved oncolytic potency. Clin Cancer Res 2001; 7(1): 120-6.

[109] Majem M, Cascallo M, Bayo-Puxan N, et al. Control of E1A under an E2F-1 promoter insulated with the myotonic dystrophy locus insulator reduces the toxicity of oncolytic adenovirus Ad-Delta24RGD. Cancer Gene Ther 2006; 13(7): 696-705.

[110] Danielsson A, Dzojic H, Nilsson B, Essand M. Increased therapeutic efficacy of the prostate-specific oncolytic adenovirus $\mathrm{Ad}[\mathrm{lsqb}] \mathrm{I} / \mathrm{PPT}-\mathrm{E} 1 \mathrm{~A}[\mathrm{rsqb}]$ by reduction of the insulator size and introduction of the full-length E3 region. Cancer Gene Ther 2008; 15(4): 203-13.

[111] Emery DW, Yannaki E, Tubbl J, et al. A chromatin insulator protects retrovirus vectors from chromosomal position effects. Proc Natl Acad Sci USA 2000; 97(16): 9150-5.

[112] Ramezani A, Hawley T, Hawley R. Performance- and safetyenhanced lentiviral vectors containing the human interferon-beta scaffold attachment region and the chicken beta-globin insulator. Blood 2003; 101(12): 4717-24.

[113] Kelly E, Russell SJ. History of oncolytic viruses: genesis to genetic engineering. Mol Ther 2007; 15(4): 651-9.

[114] Albini A, Sporn MB. The tumour microenvironment as a target for chemoprevention. Nat Rev Cancer 2007; 7(2): 139-47.

(C) Fillat et al.; Licensee Bentham Open.

This is an open access article licensed under the terms of the Creative Commons Attribution Non-Commercial License (http://creativecommons.org/licenses/by-nc/3.0/) which permits unrestricted, non-commercial use, distribution and reproduction in any medium, provided the work is properly cited. 